\title{
Deep infiltrative endometriosis with bilateral endometrioma with serous cyst adenocarcinoma in a postmenopausal woman
}

\author{
Ruby Bhatia, Sunita Mor, Bhavika Kalthe
}

Corresponding author: Dr Bhavika Kalthe, Post Graduate Student 1 $1^{\text {st }}$ year, Department of Obstetrics and Gynaecology, Maharishi Markandeshwar deemed to be university, Mullana , Ambala, Haryana, India; Email - Bhavika_kalthe@yahoo.in

Distributed under Attribution-Non Commercial - Share Alike 4.0 International (CC BY-NC-SA 4.0)

\begin{abstract}
Endometriosis is a non-neoplastic condition, although malignant transformation may occur. We refer a case of a very rare presentation in a 48 year old menopausal woman presenting with pain and feeling of heaviness in the lower abdomen, with a right adnexal mass. She was diagnosed to have deep infiltrating endometriosis grade IV with bilateral endometrioma with fibromyoma uterus with borderline serous cyst adenocarcinoma stage IB with left ovarian complex cyst. Prolonged period of infertility and chronic pelvic pain are suggestive of long standing endometriosis.
\end{abstract}

Keywords: Deep infiltrating endometriosis (DIE), epithelial ovarian malignancy, body mass index

(BMI).

Endometriosis is a condition in which there is presence of functioning endometrium (glands and stroma) in sites other than uterine mucosa. It is a common debilitating disease that occurs in 6 to $10 \%$ of general female population with peak incidence at 25-30 years ${ }^{1}$. It is a disease of the reproductive age group rarely seen before menarche and rarely after menopause. ${ }^{2}$ Incidence of endometriosis in post-menopausal women is only 3\%. Endometriosis is a non-neoplastic condition, although malignant transformation may occur. The risk of malignant transformation in ovarian endometriosis was calculated to be $2.5 \%$ but this might be underestimated. ${ }^{3}$ Epidemiological evidence from large cohort studies confirms endometriosis as an independent risk factor for ovarian cancer. Further circumstantial evidence for this link was found in the common risk factors for ovarian cancer and endometriosis. These risk factors influence retrograde menstruation and endometriosis in the same positive or negative way. The prevalence of endometriosis in epithelial ovarian cancer is around $4.5,1.4,35.9$, and $19.0 \%$ for serous, mucinous, clear-cell and endometrioid ovarian carcinoma, respectively. ${ }^{3}$

\section{Case}

A 48 year old married nulliparous post-menopausal female with obesity $(\mathrm{BMI}=27.3 \mathrm{~kg} / \mathrm{m} 2)$ was admitted with complain of pain and feeling of heaviness in the lower abdomen radiating to the lower back since 1 year. She is married for the 2nd time since 12 years and had undergone prolong treatment for infertility for around 4 years. General physical examination was satisfactory. On local abdominal examination, uterus was of 16-18weeks size of pregnant

Received: $1^{\text {st }}$ December 2019. Accepted: $17^{\text {th }}$ March 2020 .

Bhatia R, Mor S, Kalthe B. Deep infiltrative endometriosis with bilateral endometrioma with serous cyst adenocarcinoma in a postmenopausal woman. The New Indian Journal of OBGYN. 2020; 7(1): 105-7. 
uterus, lobulated abdominal mass firm in consistency, tender with irregular surface in midline. On per speculum examination cervix was normal. On bimanual pelvic examination cervix was mid positioned and a mass of 16-18 weeks size of a pregnant uterus deviated to the right side, firm in consistency, tender with irregular surface was found. Movement of the mass was transmitted to the cervix and vice versa, with restricted mobility and bilateral fornix were full and tender.

An urgent ultrasonography (USG) revealed a right adnexal complex mass with differential diagnosis of ovarian epithelial neoplasm and dermoid with multiple uterine large fibroids. The large complex solid cystic lesion was of size $8.2 \times 5.7 \mathrm{cms}$ with evidence of echogenic calcifications and internal vascularity along with thick septum within it. Upon investigation, CA-125 was increased $(86.5 \mathrm{U} / \mathrm{ml})$ with raised LDH (553 IU/L), raised TSH (9.49 uIU/ml), beta HCG was normal and normal $(13.29 \mathrm{U} / \mathrm{ml}) \mathrm{CA}-19-9$ values. Other blood counts were suggestive of anaemia $(7.6 \mathrm{mg} / \mathrm{dl})$, leucocytosis $\left(14.9 \times 10^{3} / \mathrm{cumm}\right)$ and thrombocytosis $\left(557 \times 10^{3} /\right.$ cumm $)$. Further MRI abdomen /pelvis revealed right ovarian multi cystic lesion with solid enhancing nodular components suspicious for malignant surface epithelial tumour and multiple uterine fibroids (Figure 1). A provisional diagnosis of post-menopausal, nulliparous with multiple fibromyoma with large ovarian mass of 16-18 weeks size with bilateral endometrioma with severe anaemia was made.

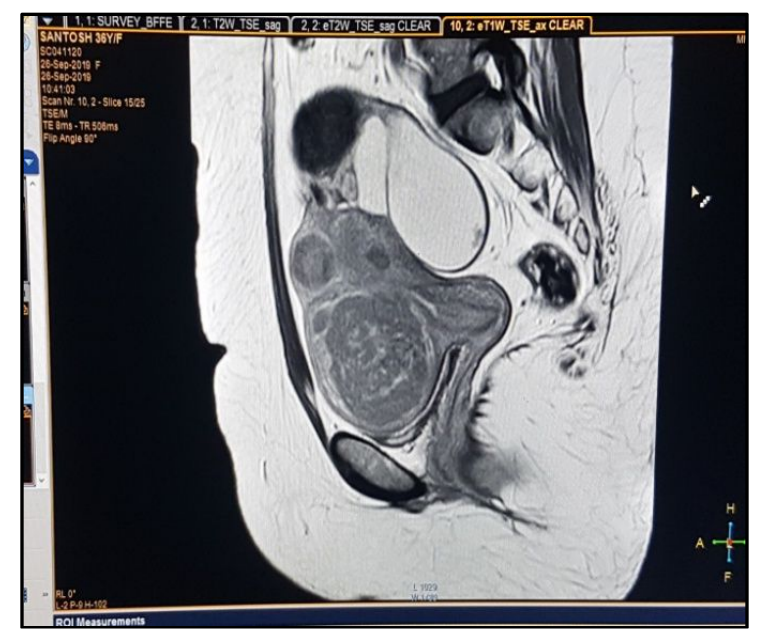

Figure 1: MRI findings

An exploratory laparotomy was performed under combine spinal epidural anaesthesia, which uterus was of 1618 weeks size and multiloculated, fixed deep down. Omentum and large gut loop were densely adherent with uterus and with bilateral endometriomas (Figure 2). There were endometriomas with appearance of multiple red flame burnt match stick and powder burns over uterus, gut, and

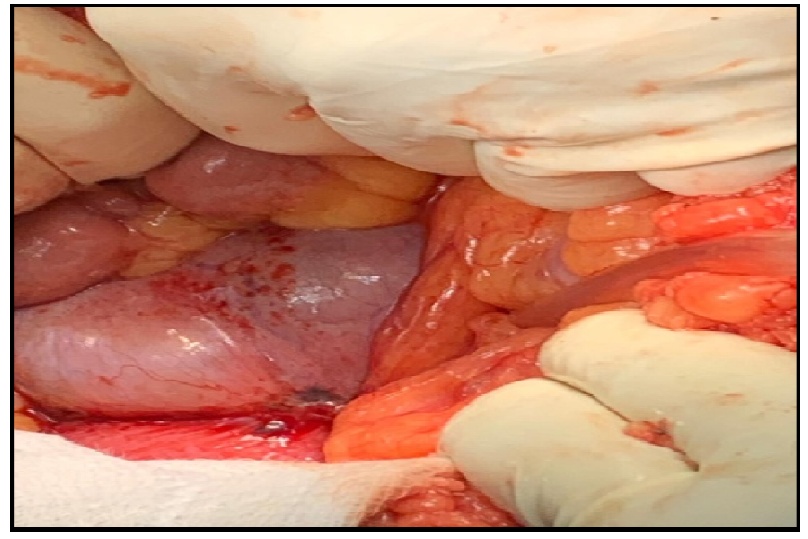

Figure 2: Intraoperative findings of fribromyoma uterus with deep infiltrating endometriosis with serous cyst adenocarcinoma showing flame shaped haemorrhage and dense adhesion to gut

lateral pelvic wall. Pouch of Douglas was obliterated with thick fibrous bands adherent with the large gut. Adhesions were separated with sharp dissection, bilateral endometriomas were excised. Frozen section confirmed borderline serous cystadenoma of left ovary. Total abdominal hysterectomy with bilateral salpingooophorectomy with infra colic omentectomy was done along with peritoneal washes. All endometriomas were ablated. Histopathology confirmed atypical proliferating serous tumour FIGO-IB with pathological staging of pT1bNxMx with endometriosis of bilateral fallopian tubes. She was managed post operatively by injection tazobactumpiperacillin 4.5 gms for 7 days. Patient was given one cycle of chemotherapy after 21 days and was called for follow up. She is doing well now.

\section{Discussion}

Studies have confirmed histologic transition from benign endometriosis to ovarian malignancy, including malignant transformation of extraovarian endometriosis. The prevalence of endometriosis in patients with epithelial ovarian cancer, especially in endometrioid and clear cell types, has been confirmed to be higher than in the general population. Ovarian cancers and adjacent endometriotic lesions have shown common genetic alterations, such as PTEN, p53, and BCL gene mutations, suggesting a possible malignant genetic transition spectrum. Furthermore, endometriosis has been associated with a chronic inflammatory state leading to cytokine release. These 
The New Indian Journal of OBGYN. 2020 (July-December); 7(1)

cytokines act in a complex system in which they induce or repress their own synthesis and can cause unregulated mitotic division, growth and differentiation, and migration or apoptosis similar to malignant mechanisms. ${ }^{4}$ Patient was not aware of any history of congestive of dysmenorrhoea, dyspareunia or dysuria. Many a times endometriosis may be asymptomatic. The malignant potential of endometriosis holds serious implications for management, such as the need for earlier and more meticulous surgical intervention for complete disease treatment.

\section{Conclusion}

Endometriosis is a disease of the reproductive age group and rarely presents in post-menopausal women. Presumptive diagnosis of endometriosis should always be kept in mind in all cases of infertility and chronic pain in reproductive years. High vigil for early diagnosis and treatment of deep infiltrating endometriosis and bilateral endometriomas early in reproductive age is need of millennium to prevent such catastrophic malignant transformation into ovarian tumours. Therefore all women with endometriosis must be screened for epithelial ovarian cancers.

\section{Conflict of interest: None. Disclaimer: Nil.}

\section{References}

1. Leibson CL, Good AE, Hass SL, Ransom, Yawn BP, Fallon WM, Melton MJ. Incidence and characterization of diagnosed endometriosis in a geographically defined population. Fertil Steril. 2004; 82(2); 314-21.

2. Brosens I, Puttemans P, Benagiano G. Endometriosis: A Life Cycle Approach? Am J Obstet Gynecol. 2013; 209(4): 307-16.

3. Jimbo H, Yoshikawa H, Onda T, Yasugi T, Sakamoto A, Taketani Y. Prevalence of ovarian endometriosis in epithelial ovarian cancer. International Journal of Gynecology \& Obstetrics. 1997; 59(3); 245-50.

4. Nezhat F, Datta MS, Hanson A, Pejovic T, Nezhat C. The relationship of endometriosis and ovarian malignancy: a review. Fertility and Sterility. 2008; 90(5); 1559-70.

Ruby Bhatia ${ }^{1}$, Sunita Mor ${ }^{2}$, Bhavika Kalthe ${ }^{3}$

${ }^{1}$ Professor and Head of Department; ${ }^{2}$ Assistant Professor; ${ }^{3}$ Post Graduate Student $1^{\text {st }}$ year, Department of Obstetrics and Gynaecology, Maharishi Markandeshwar deemed to be university, Mullana, Ambala, Haryana, India. 